\title{
THE IMPACT AND MITIGATION OF COMPLEXITY DURING PRODUCT DESIGN
}

\author{
X. ZHANG \& V. THOMSON \\ Department of Mechanical Engineering, McGill University, Canada
}

\begin{abstract}
Product complexity is driven by the interdependence of product functions, which in turn determines the interdependence of design tasks, and this is reflected in the complexity of the design process. Ever increasing product complexity has become an obstacle to effective product design. This paper introduces an agent-based model that was used to study the impact and mitigation of product complexity, where complexity was characterised by metrics defined from a knowledge perspective. In the model, a product was represented as a set of functions that required designer knowledge, component design and component integration. Designers were modelled as agents who learned knowledge through consultation and who applied knowledge to function design tasks. Variables that characterised different coordination mechanisms influenced the efficiency and quality of communication between designers and impacted the global behaviour of product design. The results from simulation experiments suggested that a growth in complexity increased effort and span time exponentially and that coordination mechanisms which quickly increased designer learning or which improved collaboration reduced overall effort. The implication for managers is that, for the design of complex products, attention should be paid to the effectiveness of coordination mechanisms, and how they reduce the time for designer learning. The implications with regard to complexity during product design can be applied to other activities where learning is a key performance factor.

Keywords: agent-based model, complexity, product design, coordination, simulation.
\end{abstract}

\section{INTRODUCTION}

Product design is the process by which a company enhances characteristics of existing products or introduces new products according to customer demand. Through this process, companies grow and adapt to evolving markets; thus, product design is essential for their viability and success [1]. Nowadays, fast-paced technology and globalisation create a very competitive market. As companies respond to challenges by increasing product diversity, introducing new technology and accelerating innovation, they are generally faced with continuously increasing complexity during product design $[2,3]$.

Product design is a complex activity that has intrinsic properties of multiplicity, dependency and uncertainty for the aspects of product and process. A product consists of numerous interconnected functions. Adding or modifying a function often involves more effort to adjust other existing functions and even requires a complete reconsideration of the whole set of product functions. Design work is usually broken down into manageable tasks where there is exchange of information in a complex network of design tasks. Since products are generally multi-disciplinary, tasks are disparate regarding knowledge base, required design tools, procedures, etc. Yet, designers are educated and trained discipline-wise [4]; so, effective inter- and intra-coordination among product functions, tasks and designers is essential for the satisfactory design and performance of products. Furthermore, 
early phases of product design often confront considerable uncertainty due to incomplete product information, limited understanding of the required functions, and a lack of both technical and design knowledge. [5]. Greater uncertainty leads to a longer design or learning cycle, making it more difficult to manage the design process. Consequently, interdependencies of product functions, coordination of these interdependencies during design activities, required interactions among designers, and system uncertainty contribute towards increasing design complexity, which becomes more challenging when shorter development times are required.

Complexity, if not well managed, is a drag on project performance and can lead to project failure. Increasing costs, longer development cycles and higher risks of failure are observed with rising complexity [6]. Case studies have shown that a company's ability to handle complexity was positively associated with the coordination of designers [7] and designers' knowledge [8]. However, these studies did not give any suggestions on how to improve team performance in projects with high levels of complexity.

The research in this paper developed an agent-based model, which enabled us to simulate the impact of product complexity on the design process and to give suggestions on which coordination mechanisms to adopt for different levels of complexity. The paper is organised as follows: section 2 defines complexity from the knowledge perspective, which serves as the basis for the model; section 3 describes the logic of the model; section 4 presents the data that served as input to the model; section 5 presents the simulation scenarios and discusses the results; and section 6 summarises the paper and gives conclusions.

\section{COMPLEXITY DEFINED IN THE KNOWLEDGE PERSPECTIVE}

Product design has been recognised as a knowledge-intensive process [9-13]. The multi-disciplinary nature of product design was found to be a key factor driving complexity [4, 14], which indicated the important contribution of designers' knowledge towards handling complexity [15]. Knowledge is the link between product and designers, as knowledge is embedded into product functions, which require designers with corresponding knowledge to realise them. Thus, we characterised product and designers from the knowledge perspective. The set of knowledge used in design projects was classified, where the levels of knowledge required for designing a function were defined with a scale: minimum (numerical value $=1$ ), general (numerical value $=2$ ) and intense (numerical value $=3$ ). Correspondingly, designers' level of ability to apply knowledge was defined with the scale: unskilled (numerical value $=1$ ), proficient (numerical value $=2$ ) and expert (numerical value = 3). Knowledge items were treated as being independent; thus, K knowledge items formed a $\mathrm{K}$-dimensional space. The knowledge of a product function or a designer was represented as a vector in the knowledge space: $\vec{V}=\left(k_{1}, k_{2}, \ldots, k_{n}, \ldots, k_{N}\right)$ where $k_{n}$ is the value of knowledge item $\mathrm{n}$ for a function or a designer.

During product design, designers do technical work to develop product functions and communicate to resolve function interfaces. The technical effort is greatly affected by the technical difficulty of functions, and the communication effort is mainly driven by interdependencies among functions. To model technical difficulty, we defined function complexity, which is the complexity of developing a certain function in the product. It is assumed that functions requiring more knowledge are more complex to design; so, the complexity of an individual function is calculated as the root mean square of the elements in its knowledge vector. To model functional interdependency, we introduced integration complexity, which is the complexity of integrating functions into a whole product. It is assumed that the integration of two functions becomes more difficult with more interfaces and with the involvement of different knowledge. Thus, the integration complexity between two functions is calculated as the product of their knowledge difference and the number of interfaces. The knowledge 
difference between two functions was defined as the intersection angle $\theta$ of their knowledge vectors. To keep the value consistent with function complexity in terms of the order of magnitude, we used $3^{\sin \theta}$ to measure the knowledge difference, since 3 was the upper limit of the knowledge scale. Defining product functions, designers and complexity from the knowledge perspective provided a basis for the development of the agent-based model of product design.

\section{AGENT-BASED MODEL OF PRODUCT DESIGN}

A research model of product design was built using the agent-based paradigm as it allowed variations in designer characteristics and in the interactions between designers. It also permitted the study of the effect of different coordination mechanisms on individuals. This model was able to track instantaneous complexity and dynamic effort during the design process.

A product was modelled as a group of functions that were knowledge intensive and interdependent. In the model, functions were agents requiring knowledge and effort to create or integrate them; designers were modelled as agents with different knowledge and experience aimed at finishing tasks such as function design, rework and functional integration. The designer agents had the autonomy to decide when to start, change or end activities such as the technical work of designing a function, consultation or coordination with another designer. Factors such as coordination efficiency, designers' experience and knowledge influenced the effectiveness and quality of communication between designers, which further determined a designer's decisions. Simultaneous design tasks as well as interactions among designer agents and function agents generated the complex behaviour of the design process, where change in effort and project duration versus different product complexity, designers' knowledge level and experience were analysed. The detailed model logic is explained in the following sub-sections.

\subsection{Technical work}

Function agents require a certain amount of technical effort determined by their function complexity. Designer agents devote effort to their assigned design tasks with different efficiency. The amount of work to do function $i$, which was denoted as $r_{i}$, was calculated with eqn (1), where $M$ was a coefficient associated with the effort estimation for the product, $W_{i}$ was the function complexity, $\mu_{p, i}$ was the technical efficiency of designer $p$ developing function $i$, and effort ${ }_{p, i}$ was the effort given by designer $p$. When $r_{i}$ was reduced to zero, the function was considered completed and ready for integration.

$$
r_{i}=M \cdot W_{i}-\sum_{p} \mu_{p, i} \cdot \text { effort }_{p, i}
$$

Technical efficiency $\mu_{p, i}$ is determined by eqn (2), where $K A_{p, k}$ is the ability level of designer $p$ applying knowledge $k, K R_{i, k}$ is the requirement level of function $i$ for knowledge $k$, and $N$ is the total number of knowledge items. It means that designers' technical efficiency increases with their level of knowledge and decreases as a function's requirement for knowledge increases.

$$
\mu_{p, i}=\frac{1}{N} \sum_{k} \frac{K A_{p, k}}{K R_{i, k}}
$$

When functions were completed, designers went to the integration phase. When designers integrated two functions, they could find incompatibility between two functions, which generated rework. It was assumed that incompatible work was more likely to happen when designers did not have enough experience on the project. A parameter, $\varepsilon_{p, i j}$, defined in the range $[0 \%, 100 \%]$ modelled a designer $p$ 's experience with the interfaces between function $i$ and function $j$. The percentage of 
technical work that needed to be reworked was determined by eqn (3), where $D_{i j}$ was the set of designers that were involved in the development of function $i$ and function $j$, and $d_{i j}$ was the number of designers in the set $D_{i j}$.

$$
\operatorname{rework}_{i j}=1-\frac{1}{d_{i j}} \cdot \sum_{p \in D_{i j}} \varepsilon_{p, i j}
$$

\subsection{Consultation on technical knowledge}

Consultation in the model was defined as communication between two designers concerning certain knowledge involved in technical work. It was modelled such that, when the knowledge requirement of a task was beyond a designer's ability, the designer consulted with a designer with a higher ability of the required knowledge. The decision to go and consult was triggered when eqn (4) was satisfied, where $K R_{i, k}$ was the required level of function $i$ for knowledge $k, K A_{p, k}$ was the ability level of designer $p$ applying knowledge $k$, and $T K$ indicated the tolerance of the knowledge gap.

$$
\frac{K R_{i, k}}{K A_{p, k}}>T K
$$

After a designer decided to consult on knowledge $k$, the designer searched for consultant designers whose knowledge $k$ was higher than or equal to the requirement of the function $\left(K A_{c n s l t, k} \geq K R_{i, k}\right)$. If there were many consultants, the designer randomly picked one of them. If no one satisfied the condition, the designer stopped looking for a consultant, and did the work at the designer's own pace.

As a result of consultation, designers' technical knowledge improved. Generally speaking, the effect of consultation was better when more consultation effort was devoted and communication was more efficient. However, consultation efficiency could be impaired by the complexity of the function. Although a designer's ability to apply knowledge improved, the improvement was constrained by the consultant's knowledge level, i.e., a designer's improved knowledge could not exceed the consultant's level of knowledge. Thus, a designer's improved ability can be quantified by eqn (5), where $K A_{p, k}^{\text {New }}$ is designer $p$ 's improved ability of knowledge $k$ after consultation, $K A_{p, k}^{\text {Old }}$ is the ability before consultation, $K A_{c n s l t, k}$ is the consultant's ability of knowledge $k$, and $c$ is the communication efficiency that can be interpreted as complexity solved per hour, $W_{i}$ is the complexity of function $i$, and effort ${ }_{p, k}$ is the consultation effort in hours. The equation means that a designer's knowledge improves faster with a more experienced consultant, and a designer cannot surpass the consultant in terms of the consulted knowledge. Also, if a designer already has a high level of knowledge before consultation, it takes less effort to reach a certain improved level. The direct effect of consultation is the improvement of a designer's ability using knowledge k. Since technical efficiency increases with designers' knowledge level according to eqn (2), the technical efficiency of developing a function where there has been consulting is improved by the effort spent on consultation.

$$
K A_{p, k}^{N e w}=K A_{c n s l t, k}-\left(K A_{c n s l t, k}-K A_{p, k}^{\text {old }}\right) \cdot e^{-\left(\frac{c \cdot e f f o r t_{p, k}}{W_{i}}\right)}
$$




\subsection{Coordination on interface}

Coordination in the model was defined as the communication between two designers who were developing different functions that were to be integrated. It was assumed that when designers were aware of a function's dependencies, they coordinated with other designers who were developing relevant functions. The decision to go and coordinate was triggered when eqn (6) was satisfied, where designer $p$ 's experience, $\varepsilon_{p, i j}$, indicated how well a designer was aware of $I_{j i}$, the integration complexity between function $i$ and function $j, c$ was the communication efficiency, effort ${ }_{p, i j}$ was the effort that designer $p$ had spent on coordinating function $i$ and function $j$, and $T I$ was the tolerance of unsolved integration complexity. After designer $\mathrm{p}$ decided to coordinate with function $j$, the designer that was assigned to function $j$ was randomly picked.

$$
\varepsilon_{p, i j} \cdot I_{i j}-c \cdot e f f o r t t_{p, i j}>T I
$$

It was assumed that a designer's experience of the interfaces between two functions, $\varepsilon_{p, i j}$, improved after coordinating the two functions. A designer's improved understanding of two functions, $\varepsilon_{p, i j}$, was determined by eqn (7), where $\varepsilon_{p, i j}^{\text {Initial }}$ was designer $p$ 's initial understanding of function $i$ and function $j, c$ was communication efficiency, $I_{i j}$ was the integration complexity of function $i$ and function $j$, and effort $t_{p, i j}$ was the effort that designer $p$ had spent on coordinating function $\mathrm{i}$ and function $\mathrm{j}$ in hours. From the equation, designer's experience improves faster with greater communication efficiency. Also, if a designer already has high experience before consultation, it takes less effort to reach a certain improved level. A designer's experience improves faster with lower integration complexity. Since rework percentage is determined by designers' experience according to eqn (3), the rework for functions that have been coordinated is reduced by the effort spent on coordination.

$$
\varepsilon_{p, i j}=1-\left(1-\varepsilon_{p, i j}^{\text {Initial }}\right) \cdot e^{-\left(\frac{c \cdot e f f o r t_{p, i j}}{I_{i j}}\right)}
$$

\section{INPUT DATA}

The model needed input data of product functions, knowledge involved in the product design, knowledge requirement of functions, interdependency among functions, and designers' ability of applying knowledge. The functional data about a hydroelectric generator was obtained from GE Hydro [16]. Through functional analysis, the product was decomposed into five major functions: control environment, provide housing, provide monitoring, provide safety and control power. The five major functions were further decomposed into sub-functions, which gave 57 functions in total.

The knowledge involved during product design was obtained by consulting the personnel who worked on the project. Ten types of knowledge were identified, including HVAC (heating, ventilating and air conditioning), air circulation, water circulation, heat transfer, electric-heat generation, control, mechanical engineering, sensor technology, physics and electrical engineering.

The personnel also gave information on the knowledge requirement for each function as well as the interdependency among functions. With the information, we were able to calculate the complexity of each function and the integration complexity between pairs of functions using the method introduced in section 2 .

The data concerning the designers in the project was unknown; so, several different scenarios were created and simulated. Twenty designer agents were created in the model to develop the product. Each designer had different ability with different knowledge. As a baseline, the designers were 
initially set as having sufficient knowledge to complete the design tasks, i.e., their knowledge matched well with the knowledge requirement of the functions. Also, they were initially set as having medium experience $(70 \%)$ about the project.

\section{SIMULATION}

The objective of the simulations was to investigate the performance of projects when different mechanisms were applied under different product complexity. By examining the chart of dynamic effort obtained from each simulation, we were able to tell how complexity unfolded with time. The chart also gave the total effort and project span time, which indicated project performance.

Factors influencing project performance in the model included: a) product complexity, b) knowledge level of designers, c) experience level of designers and d) communication efficiency. Three levels were set for each factor as illustrated below.

a) Product complexity. To obtain a series of different levels of complexity, we set the complexity level obtained from product data as the baseline and obtained a higher level of complexity by increasing the integration complexity by $50 \%$ and a lower level of complexity by decreasing the integration complexity by $50 \%$.

b) Designers' knowledge level. The level obtained from designer data was set at medium. A high level of designers' knowledge was obtained by increasing designers' knowledge level by 0.5 (but not exceeding 3, the upper limit) and a low level of designers' knowledge was obtained by decreasing designers' knowledge level by 0.5 (but not lower than 1 , the lower limit).

c) Experience in project. The baseline was $70 \%$, which meant that designers had $70 \%$ of the integration knowledge between functions. High experience was set at $95 \%$, which meant that designers were familiar with almost all the integration knowledge. Low experience was set at $45 \%$, which meant that designers knew less than half of the integration knowledge.

d) Communication efficiency. The baseline was set at 0.5 , which meant that 0.5 units of integration complexity could be resolved in one-hour of coordination. The high level was set at 0.8 and the low level at 0.2 .

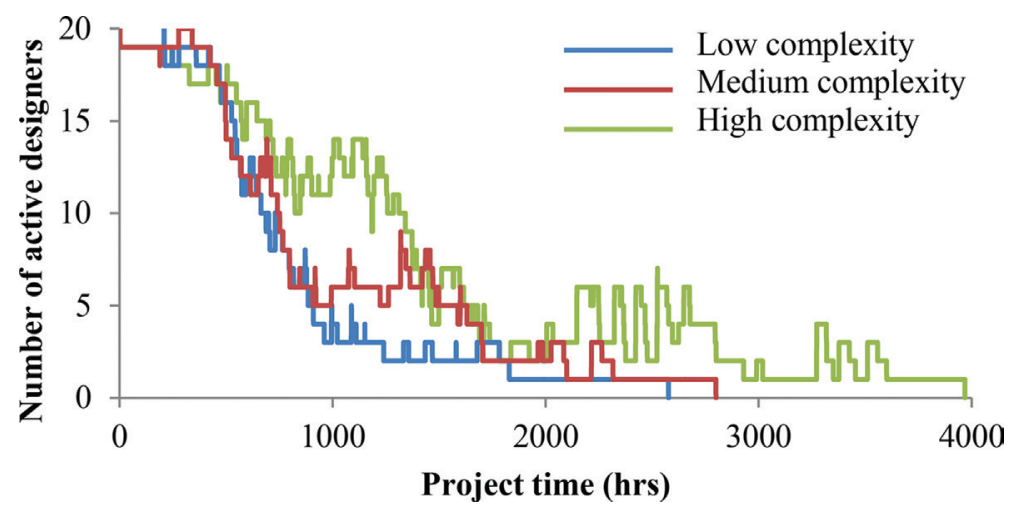

Figure 1: Effort versus project time for the design of a complex product, where complexity was low, medium or high. The data were created by modelling the activity during a multiyear project at GE Hydro. 
To study the impact of product complexity, we varied factor a) and set factors b, c and d at the medium level. To study the effectiveness of mitigation mechanisms at different levels of product complexity, we varied factors b, c and d one at a time under different levels of factor a).

\subsection{Impact of complexity}

To study the impact of complexity, designer agents, who were communicating or working on functions, were considered to be giving effort, which the model captured, and then, generated the chart of instantaneous effort during the entire project.

Figure 1 shows the effort versus project time for the design of a product at GE Hydro, where complexity was low, medium or high. The horizontal axis is project time in working hours and the vertical axis is the number of designers contributing effort at any point of time. In the case of low complexity, dynamic effort reduces continuously, which indicates that complexity is continuously being reduced and that the design problem is converging quickly. Thus, the project finishes in a relatively short time. In the case of medium complexity, dynamic effort reduces at first, increases significantly in the middle of the project, and then, decreases towards the end. When complexity is high, more frequent and large increases in effort during product design are observed. This causes the scenario to have much greater span time than the low or medium complexity case. The increase in effort in the medium and high complexity cases is due to the greater effort required to resolve issues between interdependent functions.

\subsection{Effect of mitigation mechanisms}

We studied the effect of different mitigation mechanisms on effort and project duration by varying designers' knowledge level, experience and communication efficiency under different levels of product complexity. Figure 2 compares the effect of different factors on effort and span time under different levels of product complexity. In the baseline situation, designers develop a product with medium complexity; they have moderate experience, sufficient knowledge and communicate with medium efficiency. All the other scenarios are compared with the baseline situation regarding effort and span time. The first column shows the change of effort and the second column shows the change in span time. Different rows show the scenarios varying with the different factors.

It can be seen that a higher level of designers' knowledge helps to reduce total effort and to shorten project span time. A lower level of designers' knowledge increases effort, but does not necessarily prolong the project. This can be explained by the fact that other constraints such as coordination efficiency have a large effect on project span time.

Varying the factor of experience shows that a design team with less experience is more sensitive to increasing complexity; whereas, a highly experienced design team is more stable, i.e., when developing a more complex product, the designers can control effort and span time almost to the same level as for the design of a low complexity product.

As for communication efficiency, in the case of low and medium complexity, higher efficiency does not show obvious improvement in project performance. This implies that a medium level of communication is sufficient for the design of a product with low complexity. A product with high complexity is more sensitive to the effect of communication efficiency; both effort and span time vary greatly with this factor. When communication is very efficient, effort and span time for the high complexity case can be kept at the same level as developing a product with medium complexity.

To summarise, increasing complexity requires more effort and prolongs a project, especially for innovative projects about which designers have little experience. More knowledgeable designers 
Effort
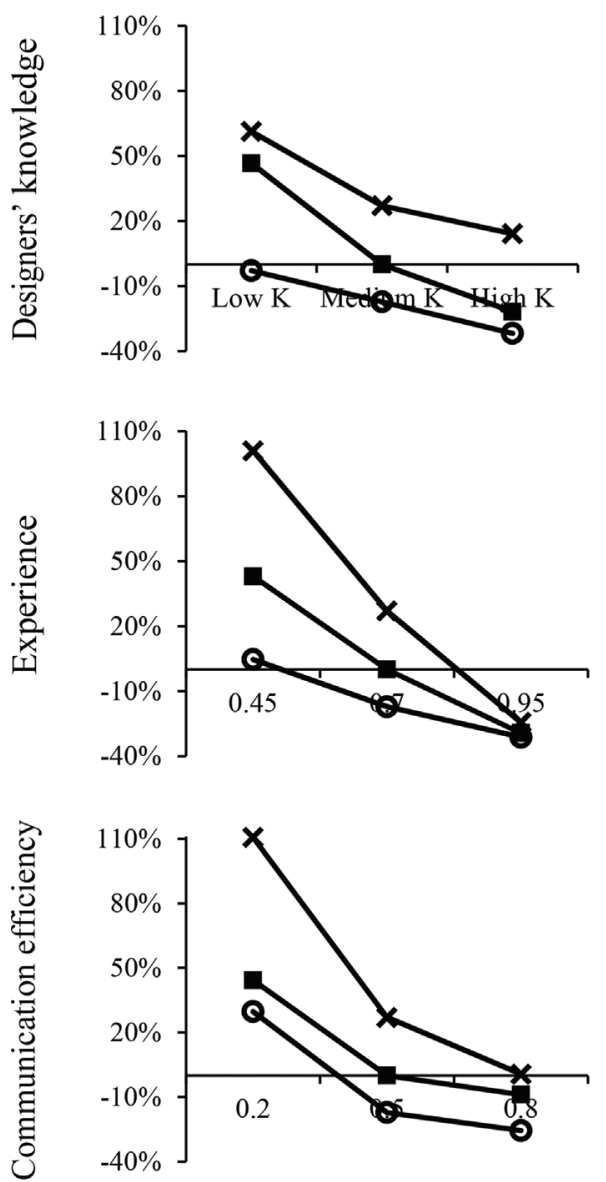

Span time
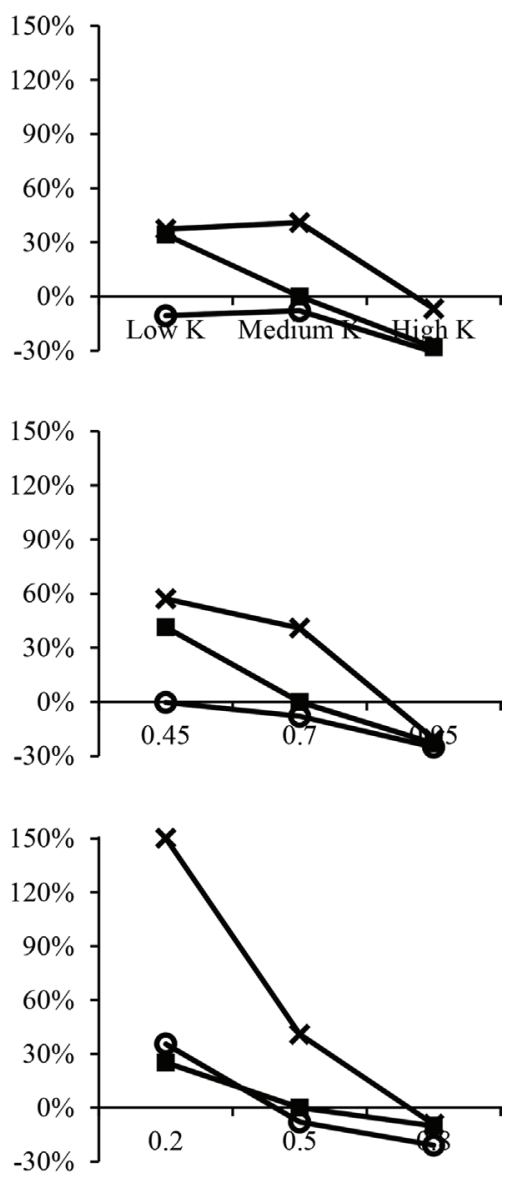

\section{- High complexity \\ - Medium complexity \\ $\times$ Low complexity}

Figure 2: The effect of different factors on effort and span time under different levels of product complexity. Effort and span time are y-axis results, and knowledge, experience and communication efficiency are $\mathrm{x}$-axis variables.

using efficient communication save effort and shorten project span time. This effect is more obvious when developing a highly complex product.

\section{CONCLUSIONS}

Ever increasing product complexity has become an obstacle to effective product design. In order to investigate how to manage projects better given greater product complexity, we defined complexity from the knowledge perspective and modelled the design process as a learning process, where designers communicate to improve knowledge about a product. Variables that characterised different mitigation mechanisms influenced the efficiency and quality of communication between designers and impacted the global behaviour of product design. The agent-based paradigm allowed us to 
model the micro-scale behaviour of designers and to observe the resultant macro-scale behaviour of the design process.

Using product data from GE Hydro, the paper simulated a series of scenarios to investigate the effect of designers' knowledge, experience and communication efficiency on project performance. Simulation results suggested that when product complexity increased, effort and span time increased. In addition, at high complexity, effort and span time increased exponentially, where the increase in effort was large and occurred towards the middle and end of a project. This increase in effort was caused by interdependency between functions. The increase in effort may not be obvious at the beginning of the project, and may be a surprise to design teams when it occurs. The simulation also showed that communication mechanisms which quickly increased designer learning or which improved collaboration reduced overall effort. The implication for managers is that, for the design of complex products, attention should be paid to the effectiveness of communication mechanisms and how they reduce the time for designer learning. Since required knowledge and learning are key factors in most projects, the implications with regard to complexity during product design can be applied to other activities where learning is a key performance factor.

\section{REFERENCES}

[1] Brown, S.L. \& Eisenhardt, K.M., Product development: past research, present findings, and future directions. The Academy of Management Review, 20, pp. 343-378, 1995.

[2] Tatikonda, M.V. \& Rosenthal, S.R., Technology novelty, project complexity, and product development project execution success: a deeper look at task uncertainty in product innovation. IEEE Transactions on Engineering Management, 47(1), pp. 74-87, 2000. http://dx.doi.org/10.1109/17.820727

[3] Lindemann, U., Maurer, M. \& Braun, T., Structural Complexity Management: An Approach for the Field of Product Design, Springer, 2008.

[4] Tomiyama, T., D’amelio, V., Urbanic, J. \& Elmaraghy, W., Complexity of multi-disciplinary design. CIRP Annals - Manufacturing Technology, 56, pp. 185-188, 2007.

[5] Oberkampf, W.L., Helton, J.C., Joslyn, C.A., Wojtkiewicz, S.F. \& Ferson, S., Challenge problems: uncertainty in system response given uncertain parameters. Reliability Engineering \& System Safety, 85, pp. 11-19, 2004.

http://dx.doi.org/10.1016/j.ress.2004.03.002

[6] Braun, S.C. \& Lindemann, U., The influence of structural complexity on product costs. IEEE International Conference on Industrial Engineering and Engineering Management, 2008. http://dx.doi.org/10.1109/ieem.2008.4737873

[7] Gokpinar, B., Hopp, W.J. \& Iravani, S.M.R., The impact of misalignment of organizational structure and product architecture on quality in complex product development. Management Science, 56, pp. 468-484, 2010.

http://dx.doi.org/10.1287/mnsc.1090.1117

[8] Erhardt, N., Is it all about teamwork? Understanding processes in team-based knowledge work. Management Learning, 42, pp. 87-112, 2011. http://dx.doi.org/10.1177/1350507610382490

[9] Nissen, M. \& Levitt, R., Toward simulation models of knowledge-intensive work processes. CIFE Working Paper, 77, 2002.

[10] Štorga, M. \& Andreasen, M.M., Complexity of product development context. DS 34: EDIProD 2004 - Fourth International Seminar and Workshop, Zielona Gora, pp. 279-284, 2004.

[11] Madhavan, R. \& Grover, R., From embedded knowledge to embodied knowledge: new product development as knowledge management. Journal of Marketing, 62, pp. 1-12, 1998. 
80 Complex Systems: Theory and Applications

http://dx.doi.org/10.2307/1252283

[12] Davis, J., Subrahmanian, E. \& Westerberg, A., Knowledge Management: Organizational and Technological Dimensions, Physica-Verlag HD, 2005.

[13] Mcgrath, J.E. \& Argote, L., Group processes in organizational contexts. Blackwell Handbook of Social Psychology: Group Processes, Blackwell Publishers Ltd., pp. 603-627, 2008.

[14] Barclay, I. \& Dann, Z., New-product-development performance evaluation: a productcomplexity-based methodology. Science Measurement and Technology, IEE Proceedings, 147, pp. 41-55, 2000.

http://dx.doi.org/10.1049/ip-smt:20000077

[15] Boisot, M., Knowledge management and complexity. The SAGE Handbook of Complexity and Management, eds. P. Allen, S. Maguire \& B. Mckelvey, SAGE Publications, 2011.

[16] Bashir, H.A. \& Thomson, V., Estimating design effort for GE hydro projects. Computers \& Industrial Engineering, 46, pp. 195-204, 2004.

http://dx.doi.org/10.1016/j.cie.2003.12.005 